\title{
The importance of Healthy Lungs in the fight against COVID-19
}

COVID-19 targets our airways and breathing systems, so it is more important than ever to look after our lung health
If our lungs are working well, they are better at keeping the airways clear, removing any harmful substances
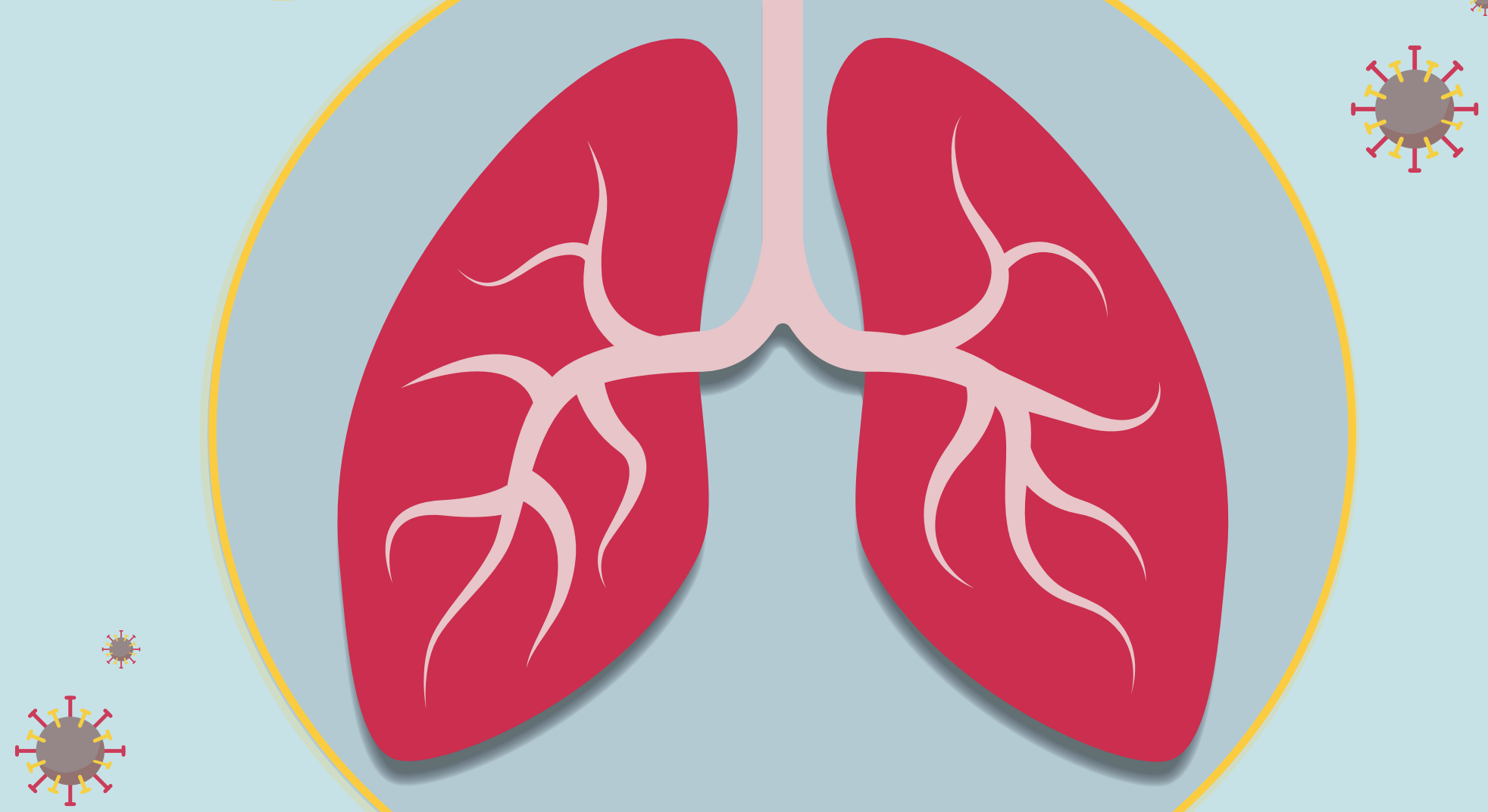

Our lungs defend us from harmful material we breathe in, such as pollution, tobacco or viruses like SARS-Cov-2, which causes COVID-19
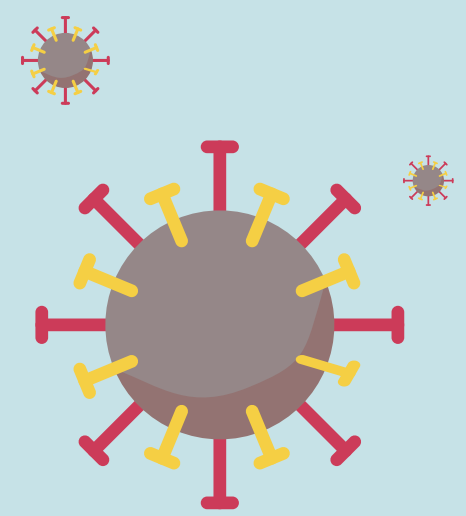

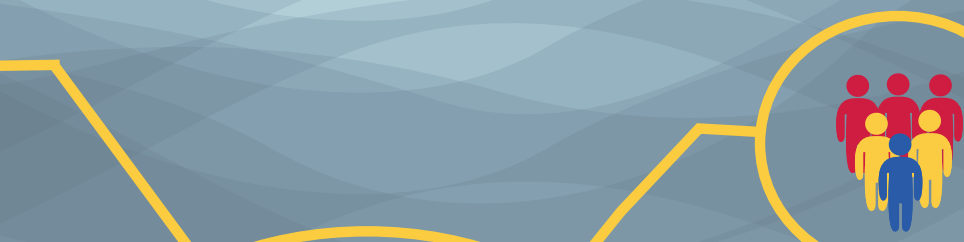

\section{1 billion}

people over the age of 14

smoke worldwide

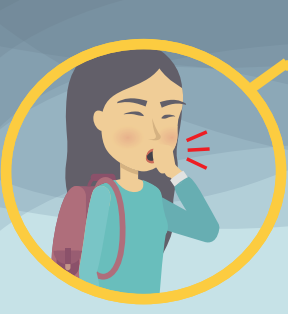

Smoking causes

airways diseases, which are a risk factor for more severe symptoms and course of COVID-19

\section{$24 h$}

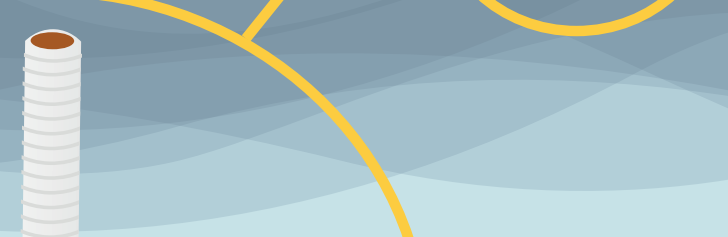

(1.)

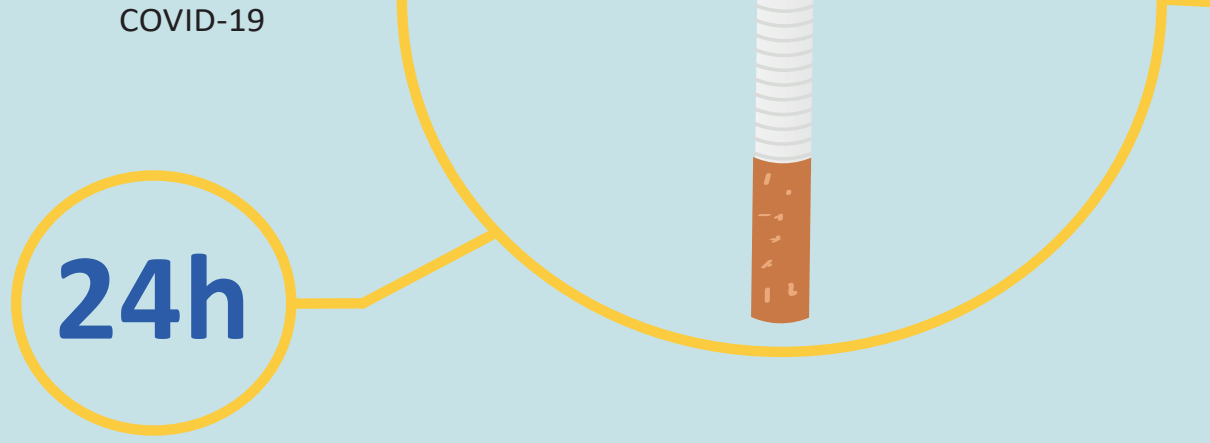

Smoking damages the lungs and makes it hard to clear out harmful substances, such as viruses and bacteria; this means that it is harder for smokers to fight off infection

Quitting smoking can help your lungs to work better within 24 hours of your last cigarette

\section{Get vaccinated}

Vaccines prevent roughly 2-3 million deaths each year

\section{1}

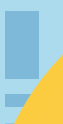

Getting vaccinated can help to keep your lungs healthy.

Viruses such as flu and pneumonia can damage the airways, increasing the risk of more severe infections and other lung diseases in the future

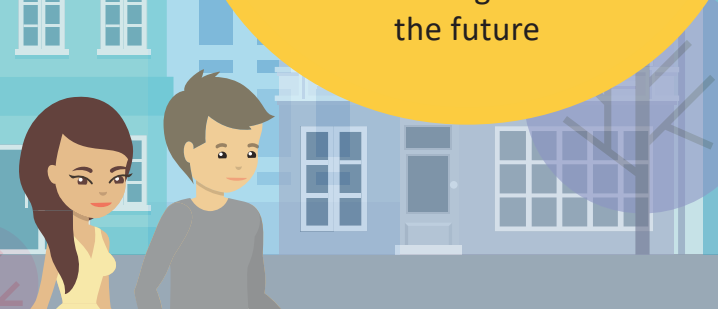

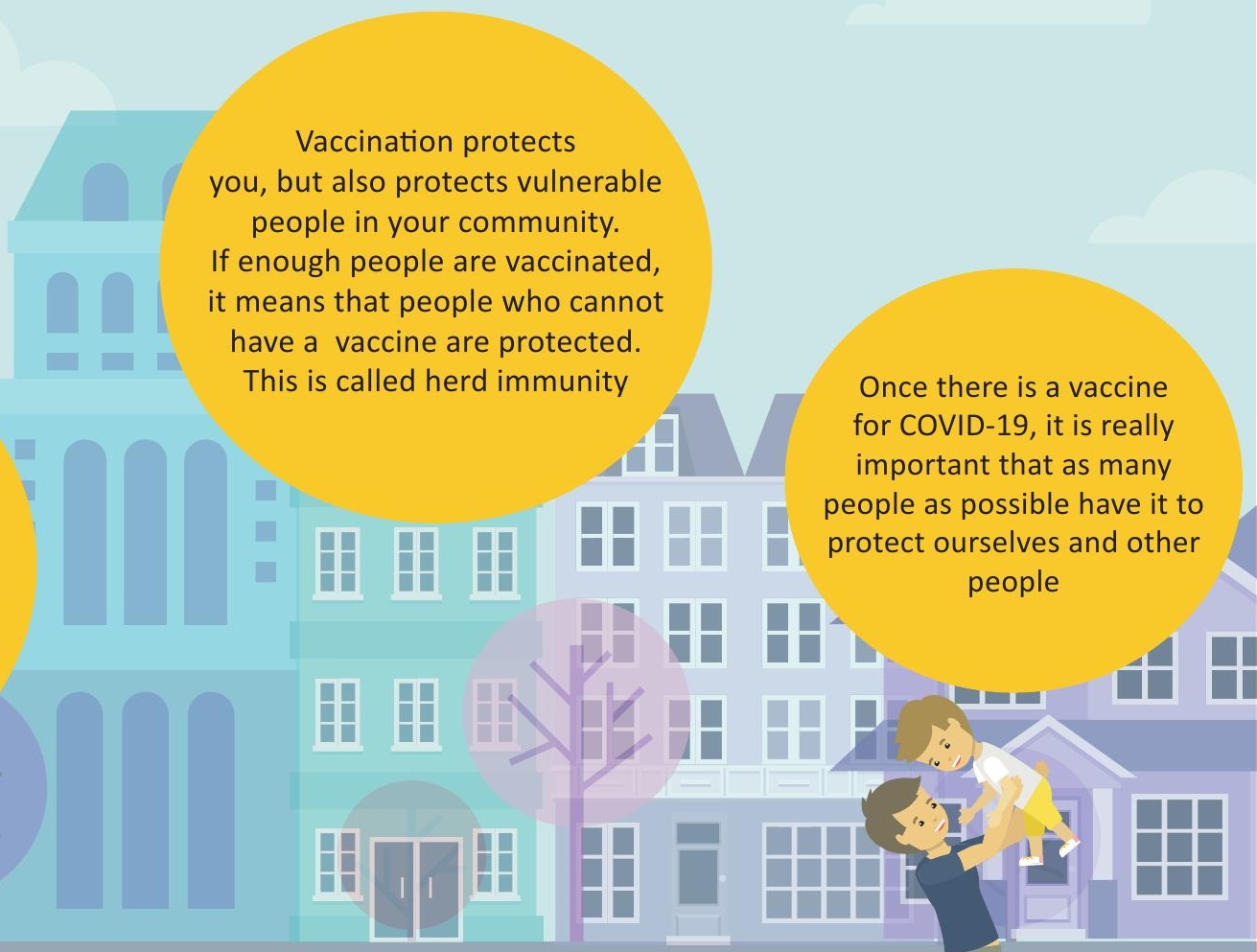




\section{Avoid air pollution}

Air pollution

can cause heart and lung diseases, stroke and diabetes - all of which are risk factors for severe symptoms of COVID-19

Long-term exposure to poor air quality can make our lungs more vulnerable to infections

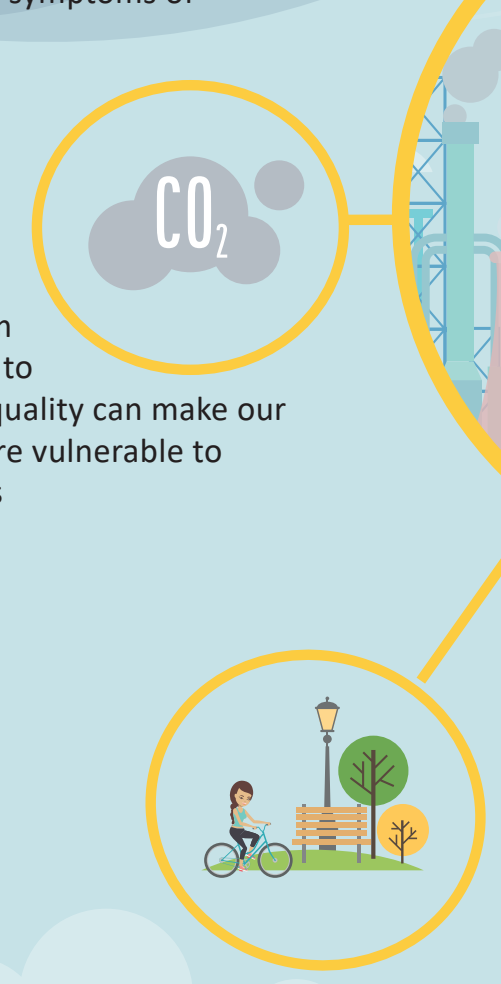

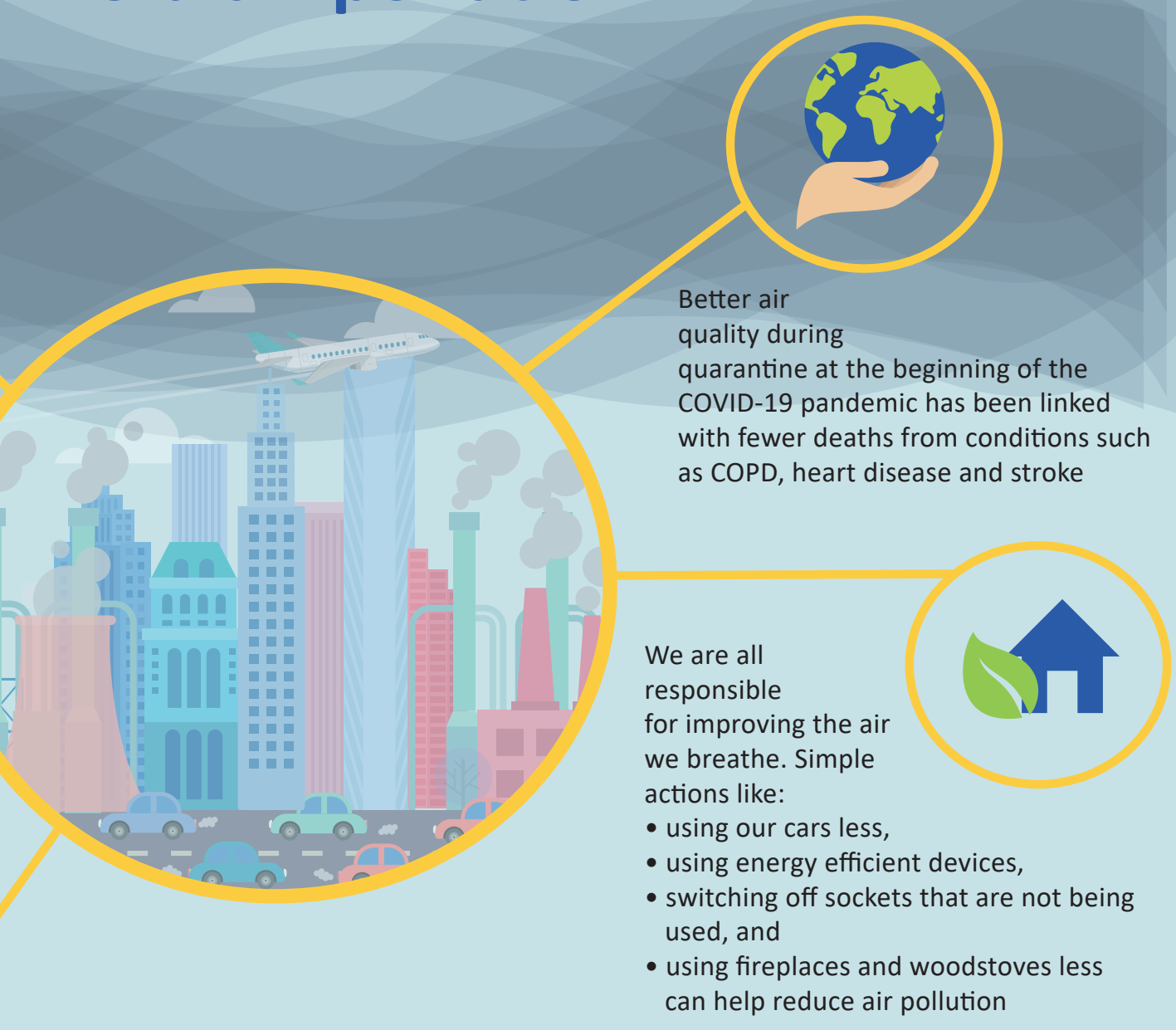

When exercising outside, walking, jogging, or cycling, try to chose routes with lower air pollution. This means smaller and less busy roads or a park

\section{Be physically active}

\section{Being physically active} can help prevent lung disease and being fit means you can recover better and faster from any infections you do catch
Walking instead of driving your car is good for you and reduces air pollution
Keeping active, even just gently at home, can improve our immune system and make it better at fighting off viruses
Regular exercise can reduce the risk of developing heart disease, obesity and diabetes, which are risk factors for severe symptoms of COVID-19 


\section{Top tips for keeping your lungs healthy}

There has never been

a more important time to quit smoking - not only for your

own health but also for those around you. Contact your local quit smoking service to get the support you need and help reduce your risk of severe symptoms of COVID-19

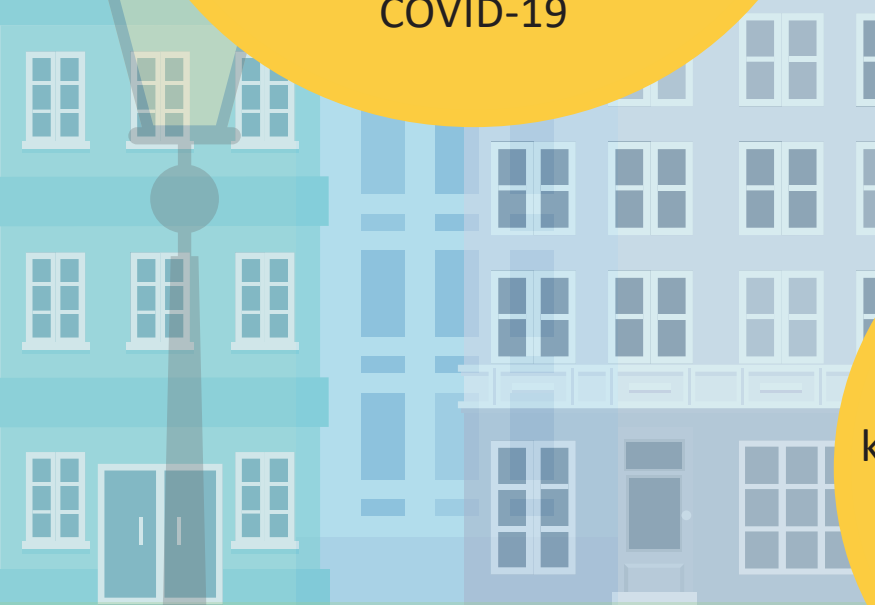

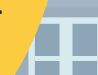

